Anton F. Guhl

\title{
Die Erfindung polytechnischer Jubiläen in der Mitte des 19. Jahrhunderts als „Verherrlichung einer großen und mächtigen Sache, einer urkräftigen Äußerung des Weltgeistes“
}

\begin{abstract}
Doing History“ is a powerful means in generating meaning and identity, particularly in uncertain settings and in times of struggle. This chapter analyzes how German-speaking polytechnics invented jubilee-tradition in the 1850s. The closer look at the anniversaries of the technical colleges in Dresden (1853), Prague (1856), and Hannover (1856) reveals a variety of media and history types that were used to assert relevance. As new institutions of higher learning the polytechnics competed with universities whose gate-keeping functions included, until 1899, the exclusive right to award doctorates. Thus, universities had a higher political and scientific standing, and their graduates enjoyed a higher societal status. Polytechnical jubilees were (also) invented to challenge this perception. By comparing the jubilee activities at the three polytechnics Dresden, Prague and Hannover, this article fosters an integrated history of higher education and adds to our knowledge of jubilee culture of non-university colleges in the $19^{\text {th }}$ century. Ultimately, it shows not only adaption and alteration of university jubilee practices but also the beginning of a specific technical jubilee culture placing alumni active in the workforce at the center of their celebrations.
\end{abstract}

\section{Einleitung}

Die inzwischen recht umfangreiche Forschung zu Hochschuljubiläen fokussiert zumeist klassische Universitäten, andere Hochschultypen spielen eine eher untergeordnete Rolle, und zur Jubiläumspraxis von Polytechnika liegen bisher keine Forschungsbeiträge vor. ${ }^{1}$ Ausgehend von dieser Forschungslücke werden in diesem Beitrag die Anfänge polytechnischer Jubiläumskultur in den 1850er Jahren anhand der drei Beispiele Prag, Dresden und Hannover betrachtet. Denn ab den

1 Siehe zum Forschungsstand zu Hochschuljubiläen den Beitrag von Anton F. Guhl und Gisela Hürlimann in diesem Band.

Ә OpenAccess. (C) 2022 Anton F. Guhl, publiziert von De Gruyter. (cc) BY Dieses Werk ist lizenziert unter einer Creative Commons Namensnennung 4.0 International Lizenz. https://doi.org/10.1515/9783110731378-005 
1850ern ließen sich die Gründungsereignisse verschiedener Polytechnika in einen dichter werdenden Jubiläumstakt einpassen. So konnten die 1806 in Prag, 1828 in Dresden und 1831 in Hannover errichteten Institutionen in der Mitte des Jahrhunderts alle einen runden Jahrestag für sich reklamieren.

Im jubiläumsfreudigen 19. Jahrhundert war der Bezug auf die Gründung ein willkommener Anlass zur Selbsthistorisierung und -darstellung. ${ }^{2}$ Im Bildungssektor nutzen nicht nur die aus dem Mittelalter überkommenen Universitäten den „Jubiläumsboom“, auch Institutionen mit weniger Gravitas feierten zum Teil umfänglich ihre Geschichte: Die Bergakademie Freiberg beispielsweise nutzte 1816 das 50. Gründungsjubiläum, um sich einen internationalen Vorbildcharakter zuzuschreiben. ${ }^{3}$ Zugleich war auch im 19. Jahrhundert ein Jubiläum kein Automatismus. Es brauchte Akteure, die sich für eine Feier einsetzten und die das Potenzial für die Deutung der Gegenwart erkannten. So verstrich etwa der 25. Gründungstag der Polytechnischen Schule in Karlsruhe im Herbst 1850 unbeachtet, und ein zufällig in jenem Jahr erschienener Bibliothekskatalog konstruierte nicht einmal aus Opportunität einen Bezug zum Jahrestag. ${ }^{4}$

Eine Betrachtung von „polytechnischen Jubiläen“ profitiert von einem Fokus auf die verschiedenen Geschichtspraktiken, die erzeugt und medial (re-)produziert wurden. Dieser Beitrag interessiert sich insbesondere für die Bezugnahmen auf universitäre Jubiläumstraditionen bei der „Erfindung“ der Jubiläen technischer Anstalten, ${ }^{5}$ für die handelnden Akteure und für deren Strategien, im Jubiläum Aufmerksamkeit herzustellen. Mit Hilfe der Selbsthistorisierung wurde nicht nur institutionelle Ambiguität und politische Unsicherheit eingehegt, sondern auch eine genuin „polytechnische“ Jubiläumskultur begründet, die deutlicher als die Universitäten die Bedeutung der (ehemaligen) Schüler für gesellschaftlichen,

2 Müller, Winfried: Inszenierte Erinnerung an welche Traditionen? Universitätsjubiläen im 19. Jahrhundert. In: Die Berliner Universität im Kontext der deutschen Universitätslandschaft nach 1800, um 1860 und um 1910. Hrsg. von Rüdiger vom Bruch unter Mitarbeit von Elisabeth Müller-Luckner. München 2010 (Schriften des Historischen Kollegs Kolloquien 76). S. 73-92, hier S. 23.

3 Albrecht, Helmuth: Die Bergakademie Freiberg. Eine Hochschulgeschichte im Spiegel ihrer Jubiläen 1765 bis 2015. Freiberg 2016.

4 Großherzogliche Badische Polytechnische Schule Karlsruhe: Catalog der Bibliothek. Aufgestellt im August 1850. Karlsruhe 1850.

5 Vgl. Wagner, Wolf Eric: Die Erfindung des Universitätsjubiläums im späten Mittelalter. In: Akademische Festkulturen vom Mittelalter bis zur Gegenwart. Zwischen Inaugurationsfeier und Fachschaftsparty. Hrsg. von Martin Kintzinger, Wolfgang Eric Wagner u. Marian Füssel. Basel 2019 (Veröffentlichungen der Gesellschaft für Universitäts- und Wissenschaftsgeschichte 15). S. 25-54. 
wirtschaftlichen und technischen Fortschritt in den Mittelpunkt rückte. ${ }^{6}$ Trotzdem verorteten sich die Polytechnika innerhalb einer gemeinsamen Hochschullandschaft mit den Universitäten. Daher folgt dieser Beitrag dem Ansatz einer integrierten Hochschulgeschichtsschreibung. Auf diese Weise können die Eigenlogiken dieser neuen, noch amorphen Anstalten adressiert werden, ohne die Gemeinsamkeiten unterschiedlicher Hochschultypen und den Transfer zwischen ihnen aus dem Blick zu verlieren. ${ }^{7}$

Die Quellengrundlage für eine Untersuchung der ersten Gründungsjubiläen der späteren Technischen Hochschulen ist heterogen, aber ergiebig. An allen drei hier fokussierten Standorten wurden Festpublikationen veröffentlicht, die - verstanden als Medien der Geschichte - einen Doppelcharakter als Primär- und Sekundärquellen aufweisen. Als in situ erzeugte Geschichtssorte sind sie Primärquellen, die Aufschlüsse über die (Jubiläums-)Akteure und die gewählten Modi zur Relevanzbehauptung aufzeigen. Zugleich dokumentieren die Dresdner und die Hannoversche Schrift die zurückliegenden Feierlichkeiten und legen trotz hagiografischer Tendenz diverse Geschichtssorten der Jubiläumsfeierlichkeiten frei. ${ }^{8}$ Weil für Dresden und Hannover keine einschlägigen Akten überliefert sind, sind die Festschriften zur Rekonstruktion der Jubiläumspraxis von zentralem Quellenwert. Im Fall von Prag gibt dagegen das dortige Hochschularchiv Einblicke in die Planung des Jubiläums, während ein erhaltener Festpokal aus Hannover auch zu Gedanken über die Materialität von Jubiläumspraktiken anregt.

Der Beitrag gliedert sich in vier Schritte. Nach einführenden Bemerkungen zur Gründungsgeschichte der feiernden Institutionen selbst geht es anschließend um die Planung der Jubiläen, die sich für Prag und Hannover nachzeichnen lassen. Denn der Akt des Planens fächert verschiedene temporale Dimensionen auf, indem die zukünftige Vergangenheit der Institution ab nun handlungsleitend wurde und auch eigene Geschichtssorten erzeugte. Die anschließend thematisierten Veranstaltungen rund um die Jubiläumsereignisse zeigen verschiedene Geschichtspraktiken, bei denen der Kreis der Jubiläumsakteure ebenso heterogen ist wie die mediale Inszenierung von Geschichte. Am nachhaltigsten greifbar bleiben die Festschriften, die daher besonders adressiert und hinsichtlich der verschiedenen Modi der Relevanzzuschreibung untersucht werden.

6 Vgl. zur akademischen ,invention of tradition“ Paletschek, Sylvia: Die permanente Erfindung einer Tradition. Die Universität Tübingen im Kaiserreich und in der Weimarer Republik. Stuttgart 2001 (Contubernium. Tübinger Beiträge zur Universitäts- und Wissenschaftsgeschichte 53).

7 Guhl, Anton F.: Perspektiven einer integrierten Hochschulgeschichte. In: Jahrbuch für Universitätsgeschichte 23 (2020) [im Druck].

8 Logge, Thorsten: Geschichtssorten als Gegenstand einer forschungsorientierten Public History. In: Public History Weekly 6:24 (2018). 


\section{Zur Gründung von Polytechnika}

Im Zuge der Umwälzungsprozesse zwischen 1789 und 1815 wurde im Alten Reich zunächst etwa jede zweite Universität geschlossen. ${ }^{9}$ Neben der allgemeinen wurde in diesen Jahren zugleich die höhere technische Bildung reorganisiert. Nach der 1794 in Paris gegründeten École Polytechnique wurden zunächst in Prag (1806), Wien (1815) und Karlsruhe (1825) Polytechnika ins Leben gerufen. ${ }^{10}$

Die neugegründeten Polytechnischen Schulen und Institute - von denen viele später zu Technischen Hochschulen und Technischen Universitäten wurden entsprachen in vielerlei Hinsicht weder den damaligen Universitäten noch den späteren Technischen Hochschulen. ${ }^{11}$ Trotz der Unterschiede zwischen Polytechnika und Universitäten ist es auffällig, dass es auf der Ebene der Lernenden früh zu einem Transfer sozialer Praktiken kam: Erste „Studentenverbindungen“ bildeten sich Ende der 1830er Jahre. ${ }^{12}$ Die Lehrer und Professoren der Polytechnika entstammten entweder dem höheren Schulwesen, den Fachschulen vor Ort oder waren an Universitäten tätig, sodass sich auch bei ihnen zahlreiche Verbindungslinien innerhalb der Hochschullandschaft zeigen lassen.

9 Kraus, Hans-Christoph: Kultur, Bildung und Wissenschaft im 19. Jahrhundert. München 2008 (Enzyklopädie deutscher Geschichte 82). S. 22.

10 Guhl, Anton F.: Technik als blinder Fleck der Universitätsgeschichte? Die Debatte um die Gründung von Polytechnika Anfang des 19. Jahrhunderts und ihre Ausblendung durch die Universitätsgeschichte. In: Hochschulen im öffentlichen Raum. Historiographische und systematische Perspektiven auf ein Beziehungsgeflecht. Hrsg. von Martin Göllnitz u. Kim Krämer. Göttingen 2020 (Beiträge zur Geschichte der Universität Mainz Neue Folge 17). S. 81-99; vgl. noch immer Manegold, Karl-Heinz: Universität, Technische Hochschule und Industrie. Ein Beitrag zur Emanzipation der Technik im 19. Jahrhundert unter besonderer Berücksichtigung der Bestrebungen Felix Kleins. Berlin 1970 (Schriften zur Wirtschafts- und Sozialgeschichte 16); ders.: Geschichte der Technischen Hochschulen. In: Technik und Bildung. Hrsg. von Laetitia Boehm u. Charlotte Schönbeck. Düsseldorf 1989 (Technik und Kultur 5). S. 204-234; König, Wolfgang: Stand und Aufgaben der Forschung zur Geschichte der deutschen Polytechnischen Schulen und Technischen Hochschulen im 19. Jahrhundert. In: Technikgeschichte 48:1 (1981). S. 47-67; ders.: Spezialisierung und Bildungsanspruch. Zur Geschichte der Technischen Hochschulen im 19. und 20. Jahrhundert. In: Berichte zur Wissenschaftsgeschichte 11 (1988). S. 219-225.

11 König, Wolfgang: Zwischen Verwaltungsstaat und Industriegesellschaft. Die Gründung höherer technischer Bildungsstätten in Deutschland in den ersten Jahrzehnten des 19. Jahrhunderts. In: Berichte zur Wissenschaftsgeschichte 21 (1998). S. 115-122.

12 Grobe, Frank: Die technischen Burschenschaften. In: „Deutschland immer gedient zu haben ist unser höchstes Lob“ - Zweihundert Jahre Deutsche Burschenschaften. Hrsg. von Harald Lönnecker. Heidelberg 2015 (Darstellungen und Quellen zur Geschichte der deutschen Einheitsbewegung im neunzehnten und zwanzigsten Jahrhundert 21). S. 701-780. 
Bereits die Gründung der Polytechnika wurde von ausführlichen öffentlichen Debatten begleitet, in denen vor allem die Pariser Anstalt ,in vielen deutschen gelehrten Schriften, Journalen und Zeitungen [...] als Muster vielversprechender Einrichtungen“ galt. ${ }^{13}$ Die „Lebensäußerung der Institution“ in den JubiläumsFesten und -Schriften baute somit auf einem seit Jahrzehnten virulenten Diskurs auf, ${ }^{14}$ der von heterogenen Beiträgen geprägt war. ${ }^{15}$

Die neuen technischen Lehranstalten unterschieden sich zu Beginn erheblich, und auch die drei Jubilarinnen wiesen Unterschiede auf. Das 1806 gegründete Prager Polytechnikum, das erste im deutschsprachigen Raum, konnte 1856 bereits seine 50-Jahr-Feier abhalten. Maßgeblich auf Betreiben der Böhmischen Stände ins Leben gerufen, war das „ständisch polytechnische Institut zu Prag“ somit auch älter als die Wiener Schwesteranstalt, die aber mit dem Moment ihrer Gründung im Jahr 1815 das Prager Institut dauerhaft in den Schatten stellen sollte, was nicht ohne Rivalität und Animositäten einherging. ${ }^{16}$ Auch im Jubiläum und der bei dieser Gelegenheit vorgelegten Festschrift wird diese Nachrangigkeit zum Thema. Die Dresdner Einrichtung wurde 1828 als technische Bildungsanstalt mit praktischem Schwerpunkt begründet und erst 1851 in den Rang eines Polytechnikums erhoben. ${ }^{17}$ Auch wenn das Wiener Polytechnische Institut als Vorbild diente, blieb es unerreicht. Die Anstalt in Hannover war 1831 als höhere Gewerbeschule gegründet worden und wurde 1847 durch die Erweiterung bauinge-

13 Gerstner, Franz Joseph: Über die polytechnische Lehranstalt. In: Nachrichten von der beabsichtigten Verbesserung des öffentlichen Unterrichtswesens in den österreichischen Staaten mit authentischen Belegen. Hrsg. von Christian Ulrich Detlev Eggers. Tübingen 1808. S. 328-365, hier S. 335 .

14 Eckardt, Hans Wilhelm: Akademische Feiern als Selbstdarstellung der Hamburger Universität im „Dritten Reich“. In: Hochschulalltag im „Dritten Reich“. Die Hamburger Universität 1933-1945. 3 Teile. Hrsg. von Eckart Krause, Holger Fischer u. Ludwig Huber. Berlin/Hamburg 1991 (Hamburger Beiträge zur Wissenschaftsgeschichte 3). S. 179-200, hier S. 179.

15 Vgl. als pars pro toto Gerstner, Lehranstalt (wie Anm. 13); Prechtl, Johann Joseph (Hrsg.): Jahrbücher des Kaiserlichen Königlichen Polytechnischen Institutes in Wien. Wien 1819-1839; Köhler, Heinrich Gottlieb: Über die zweckmäßige Einrichtung der Gewerbsschulen und der polytechnischen Institute. Göttingen 1830; Romberg, Johann Andreas: Entwurf zur polytechnischen Schule in Hamburg. Hamburg 1835; Schoedler, Friedrich: Die höheren technischen Schulen nach ihrer Idee und Bedeutung dargestellt und erläutert durch die Beschreibung der höheren technischen Lehranstalten zu Augsburg, Braunschweig, Carlsruhe, Cassel, Darmstadt, Dresden, München, Prag, Stuttgart und Wien. Braunschweig 1847; o.V.: Ueber die Erweiterung der staatswirthschaftlichen Facultät. In: Deutsche Universitäts-Zeitung. Centralorgan für die Gesammtinteressen deutscher Universitäten Nr. 23 (6.6.1849).

16 Vgl. noch immer Neuwirth, Joseph (Hrsg.): Die K.K. Technische Hochschule in Wien 18151915. Gedenkschrift. Wien 1915; siehe auch den Beitrag von Juliane Mikoletzky in diesem Band. 17 Pommerin, Reiner: Geschichte der TU Dresden 1828-2009. Köln/Weimar/Wien 2003. S. 5-19. 
nieurtechnischer Inhalte zum Polytechnikum. ${ }^{18}$ Der Wiener Einfluss war in Hannover ebenfalls zentral, zumal der Gründungsdirektor Karl Karmarsch (18031879) am Wiener Polytechnikum studiert hatte. Karmarschs rege Publikationstätigkeit zu Werdung und Entwicklung des Hannoverschen Polytechnikums zeigt sein Streben, die Anstalt in die öffentliche Diskussion zu bringen. ${ }^{19}$ Eine publizistische Nutzbarmachung des Jahrestags war nur folgerichtig.

\section{Die zukünftige Vergangenheit - Jubiläumsplanungen}

In der Vorbereitung der Jubiläen verbinden sich eindrücklich die ohnehin bestehenden temporalen Verschränkungen zwischen Erinnern, Gegenwart und erhoffter Zukunft. Mit einer „Futur-II-Brille“ wurde das Jubiläum gedanklich vorausgenommen, und die zukünftige Vergangenheit strukturierte das Handeln. ${ }^{20}$ Tatsächlich gelang es dem Prager Polytechnikum pünktlich zum Jahrestag, eine 400-seitige Festschrift vorzulegen, doch täuscht der Eindruck, dieser Erfolg sei auch mit einer langfristigen und sorgfältigen Planung einhergegangen. Denn erst ein halbes Jahr vor dem Festakt hatte sich der Direktor des Prager Polytechnikums Josef Thaddäus Lumbe (1801-1879) an den ständischen Landesausschuss gewandt und um Genehmigung und Finanzierung gebeten. ${ }^{21}$ Dabei stellte Lumbe das Jubiläum seiner Institution in einen Kontext mit dem 1848 gefeierten 500jährigen Jubiläum der Prager Universität. Den Bedeutungsvorsprung der Universität erkannte Lumbe an, und es könne „nicht in der Absicht des Lehrkörpers liegen[,] eine auch nur einigermaßen ähnliche Feier [...] zu veranstalten“, um gleichzeitig festzuhalten, dass Beispiele vorlägen, wonach „selbst ein 25jähri-

18 Kokkelink, Günther: Polytechnische Lehranstalt im Königreich Hannover - von den Anfängen bis in die zwanziger Jahre. In: Die Universität Hannover. Ihre Bauten, ihre Gärten, ihre Planungsgeschichte. Hrsg. von Sid Auffarth u. Wolfgang Pietsch. Petersberg 2003. S. 65-93.

19 Karmarsch, Karl: Die höhere Gewerbeschule in Hannover. Erläuterungen über Zweck, Einrichtung und Nutzen derselben. Hannover 1831; ders.: Die höhere Gewerbeschule in Hannover. 2. sehr erweiterte Aufl. Hannover 1844; ders.: Die Polytechnische Schule zu Hannover. Hannover 1848.

20 Mischner, Sabine: Zeitregime des Krieges: Zeitpraktiken im Ersten Weltkrieg. In: Die Zukunft des 20. Jahrhunderts. Dimensionen einer historischen Zukunftsforschung. Hrsg. von Lucian Hölscher. Frankfurt am Main 2017. S. 75-100, hier S. 85.

21 Hier und im Folgenden: Archiv der Tschechischen technischen Universität (CVUT), Polytechnický ústav: Lumbe an Ständischen Landesausschuss, 17.6.1856. 
ges Gründungsfest technischer Schulen in Deutschland [...] feierlich begangen wurde“.

Beide Verweise sind zentral für die Selbstverortung des Prager Polytechnikums. Als höhere (Aus-)Bildungsanstalt in Prag liegt erstens der Vergleich mit der altehrwürdigen Universität auf der Hand, wenngleich (noch) jede Konkurrenz vermieden wird. Der Blick nach Deutschland auf Jubiläen dortiger technischer Schulen zeigt zweitens, dass die Tradierung der eigenen Geschichte auch und gerade im Vergleich mit anderen Polytechnika angestrebt wurde. Schließlich sei man es, „der hohen Bedeutung des technischen Instituts schuldig [...], die Gönner[,] Freunde und Schüler der Anstalt und die Schwesteranstalten des Innund Auslandes auf den langen Bestand derselben aufmerksam“ zu machen. Die Antwort des Landesausschusses war zögerlich. Unmissverständlich wurde beschieden, vor einer Entscheidung habe „das Direktorat zuvor noch den beiläufigen Kostenbetrag unter näherer Auseinandersetzung des Festprogrammes zu praeliminieren“. 22

Aufgrund der fortgeschrittenen Zeit konnte Lumbe nicht auf die Genehmigung warten und schritt in den Vorbereitungen der Feier voran. Das Polytechnikum bemühte sich schon bald darum, den Verbleib seiner Schüler zu erkunden. In der Presse erschienen kurze Notizen mit der Aufforderung an ehemalige Schüler, sich zu melden. ${ }^{23}$ In anderen Zeitungen erschienen Texte mit demselben Appell, die über eine bloße Recherche hinausgingen und als eigenständige jubiläumsbasierte Geschichtssorte gelten können:

„Das polytechnische Institut zu Prag [...] das älteste, in Österreich und ganz Deutschland, feiert noch in diesem Jahre die fünfzigste Jahreswiederkehr seiner Eröffnung in seiner gegenwärtigen Gestalt. In diesen 50 Jahren ist eine große Anzahl von Männern aus demselben hervorgegangen, welche demselben ihre geistige Ausbildung verdanken und sich dadurch meist zu gesicherten und ehrenvollen Stellungen in der bürgerlichen Gesellschaft emporgeschwungen haben. Das geistige Band aber, welches eine Bildungsanstalt mit ihren Zöglingen verknüpft, ist mit dem Austritte der letzteren aus der Anstalt nicht gelöst.“24

22 CVUT, Polytechnický ústav: Ständischer Landesausschuss an das Direktorat des ständischtechnischen Instituts, 18.7.1856.

23 Vgl. Fremden-Blatt [Wien] (8.8.1856.). o.S.; desgleichen: Troppauer Zeitung Nr. 185 (10.8. 1856). o.S.

24 Österreichisch-kaiserliche Wiener Zeitung Nr. 187 (13.8.1856). S. 2388; ebenfalls in: Laibacher Zeitung Nr. 189 (18. 8.1856). S. 822. 
Am 15. September 1856 schließlich übermittelte Lumbe das vorläufige Programm und den Kostenüberschlag an den Landesausschuss. ${ }^{25}$ Für das 50. Jubiläum des Gründungstags, den 10. November 1856, war ein feierlicher Gottesdienst in der Pfarrkirche St. Egyd geplant und eine Versammlung nach dem Gottesdienst in einem festlich geschmückten Saal des Instituts, „wobei auf die Feier bezügliche Reden gehalten würden“. Als bleibendes Denkmal dienten die Herausgabe der Festschrift und die Begründung eines Stipendiums. Hier orientierte sich Lumbe explizit an dem „Vorgange des Lehrercollegiums der Dresdener polytechnischen Schule“. Die nötigen Mittel für das Stipendium sollten durch Subskriptionen für die Festschrift gewonnen werden, denn die eigentlichen Druckkosten sollte der Landesausschuss tragen. Überdies war ein (allerdings privat finanziertes) Festessen vorgesehen, um in der „Verbindung von Institut und praktischen Berufskreisen eine neuere Berührung zu erzielen“.

Die abhängige Stellung des Direktors nicht nur, aber auch in der Begründung von Jubiläumspraktiken wird aus dessen Erklärung ersichtlich, „sich auf das bereitwilligste allen höheren Andeutungen [zu] fügen“, falls der Landesausschuss „eine Änderung oder Bereicherung des Programmes“ wünsche. Die Kosten der Feier taxierte Lumbe auf 771 Gulden und verschätzte sich damit erheblich. Vor allem die Festschrift sollte teurer werden als erwartet, denn der Umfang wuchs von geplanten 15 Bogen auf 23, und statt 1.000 Exemplaren wurden in Folge der zahlreichen Subskriptionen 2.040 Stück aufgelegt. Am Ende beliefen sich die Jubiläumskosten „durch das Zusammentreffen mannigfaltiger Umstände trotz der angestrebten höchsten Sparsamkeit“ auf knapp 2000 Gulden, von denen allein die Festschrift mit 1367 Gulden zu Buche schlug. ${ }^{26}$

Erheblich stiegen auch die Kosten für die Dekoration des Saals, für die eigens ein Portrait des Gründungsdirektors Franz Joseph von Gerstner (1756-1832) aus dessen Geburtsstadt Komotau ausgeliehen und mit einem zweitägigen FuhrwerkTransport nach Prag verbracht wurde. Die bereitwillige Leihgabe zeigt die Interkonnektivität der verschiedenen Geschichtssorten Festakt und Portraitmalerei oder anders ausgedrückt: Der Vorgang verdeutlicht, wie ein externer Akteur (die Stadtbehörden von Komotau) das Jubiläum für seine eigene Geschichtserzählung nutzt, um einen Teil der Aufmerksamkeit für das Polytechnikum auch auf Komotau zu lenken, wo Gerstner „in solcher Achtung steht [...], daß seinem Portrait in dem früheren Magistratssitzungssaale zur Bewahrung seines immerwährenden Andenkens ein Ehrenplatz angewiesen war“ ${ }^{27}$

25 Hier und im Folgenden CVUT, Polytechnický ústav: Lumbe an ständischen Landesausschuss, 15.9.1856 [Hervorhebung im Original].

26 CVUT, Polytechnický ústav: Lumbe: Bericht über die Ausgaben für die Feier, 16.1.1857.

27 CVUT, Polytechnický ústav: Stadt Komotau an Direktorat, 28.10.1856. 


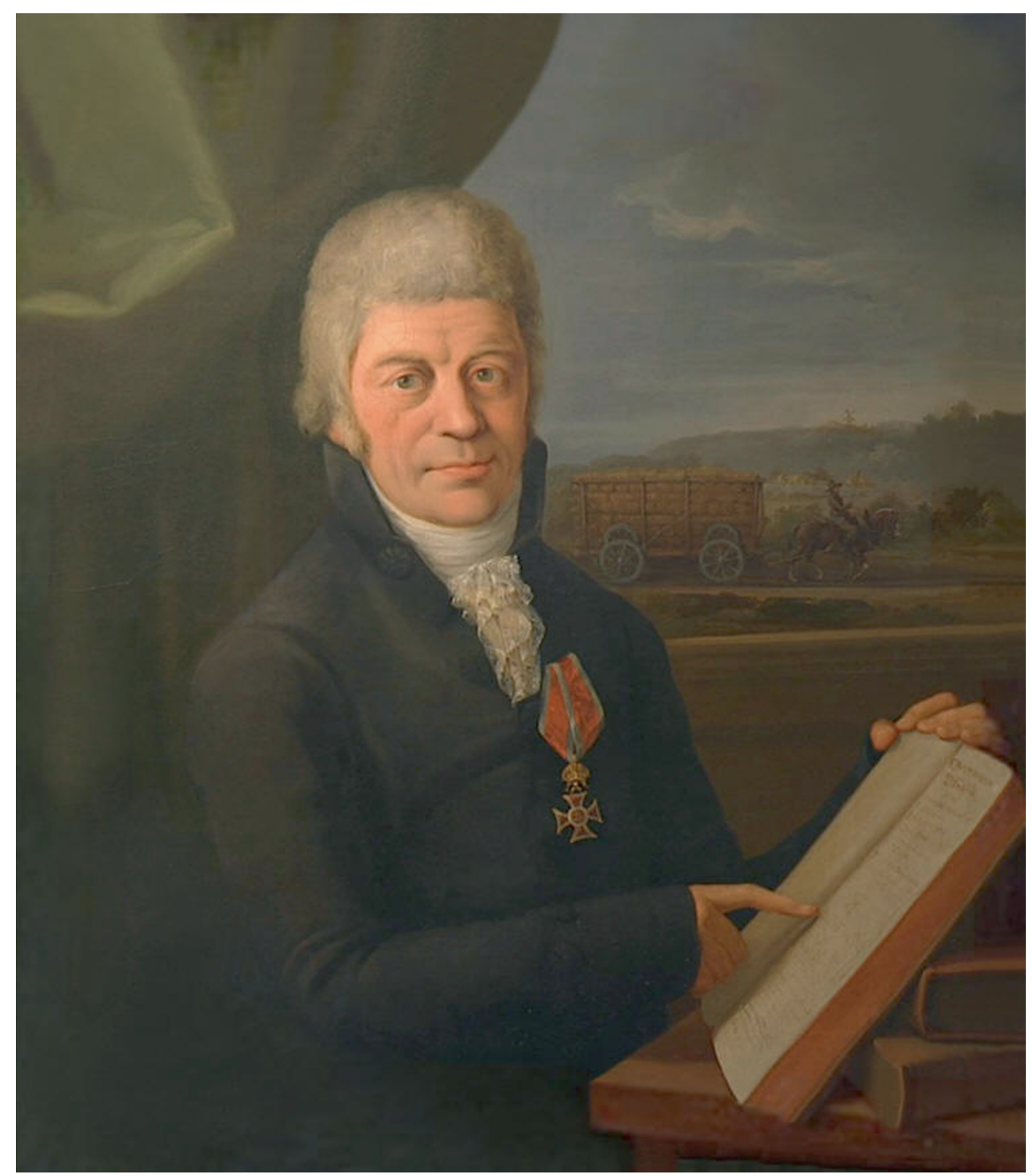

Abb. 1: Der Gründungsdirektor weist dem Prager Polytechnikum den Weg. Franz Joseph von Gerstner portraitiert von Josef Bergler (1815).

Während die Planungen für das Dresdner Jubiläum im Dunkel bleiben, sind die Vorbereitungen zur Feier des Polytechnikums in Hannover dezidierter Bestandteil der dortigen Festschrift. Der ehemalige Schüler der Anstalt und nunmehrige Eisenbahnbau-Inspektor Ernst Friedrich Buresch (1817-1892) leitete den Bericht des Hannoveraner Jubiläums mit dessen Planung ein. So diente die Festschrift nicht nur der Dokumentation der zurückliegenden Feier, sondern auch ihrer sorgfältigen Vorbereitung, also ihrer Vorvergangenheit. Ein Festkomi- 
tee wurde gebildet, das vor allem aus ehemaligen Schülern getragen wurde, drei von ihnen lehrten zu jenem Zeitpunkt am Polytechnikum. Die Ernsthaftigkeit, mit der die Sache angegangen wurde, zeigt die Wahl eines Vorsitzenden (Konrad Wilhelm Hase, 1818-1902), eines Schriftführers (Ludwig Debo, 1818-1905) sowie eines Kassenführers (Franz Beckmann, 1811-1876). Von Beginn an wurde das Fest als standespolitische Chance erkannt und in „regelmäßig und zahlreich gehaltenen Sitzungen“ setzte sich die einstimmige Ansicht durch, „daß die projektierte Feier eine durchaus ernste, würdige und dabei großartige sein müsse“. ${ }^{28}$ Das Festkomitee wollte mit den Festivitäten, „die Wichtigkeit und den gegenwärtigen hohen Standpunkt der polytechnischen Wissenschaften und Künste überhaupt“ ausdrücken und auch „Denjenigen (!) einleuchtend“ machen, die darüber „noch in Unkenntnis oder Vorurtheil befangen sein möchten“.

Von den über 2000 Alumni der Schule brachte das emsige Komitee immerhin von etwa der Hälfte den Aufenthaltsort in Erfahrung und schickte ihnen Einladungen. Die Jubiläumseinladung umfasste auch einen vom Bibliothekar des Polytechnikums, Ernst Rommel (1819-1892), verfassten „Festgruß“ mit einem Loblied auf die Natur- und Technikwissenschaften. ${ }^{29}$ In der sehr genauen Darstellung der „vielfachen Arbeiten“ des Komitees (bis hin zur Zahl der unzustellbaren Einladungen) setzte Buresch den Jubiläumsakteuren selbst ein Denkmal und dokumentierte auch, wer sich in den Subkomitees für den Ball und den Festzug aus ehemaligen und aktuellen Schülern der Anstalt engagierte. ${ }^{30}$

Bureschs Darstellung vermittelt den Eindruck, als habe die Jubiläumsplanung das gesamte Polytechnikum erfasst, wo gleich mehrere Räume in Fest-Ateliers umgewandelt wurden, in denen ,wochenlang die rührigste Thätigkeit herrschte“. An den Vorbereitungen waren auch Frauen zentral eingebunden, obgleich ihnen der Besuch der Anstalt noch für Generationen verwehrt bleiben sollte; ein „Damen-Verein unter den Auspizien der Frau Professorin Kühlmann“ fertigte „die für den Festzug erforderlichen zahlreichen Banner, Fähnlein, Schärpen und sonstigen Dekorations- und Kostümgegenstände“. Auch Konflikte in der Planungsphase werden zumindest angesprochen. So fand sich zehn Tage vor dem Anlass das bisherige Festzugskomitee „bewogen“, seinen „Auftrag zurückzugeben und sich aufzulösen“, sodass rasch ein Ersatzkomitee gebildet werden musste.

28 Hier und im Folgenden Buresch, Ernst Friedrich: Die Feier des 25jährigen Bestehens der polytechnischen Schule, am 2. Mai 1856. In: Karmarsch, Karl: Die polytechnische Schule zu Hannover. 2., sehr erweiterte Aufl. Hannover 1856. S. 189-208, hier S. 190.

29 Rommel, Ernst: Festgruß zum fünfundzwanzigjährigen Jubiläum der polytechnischen Schule zu Hannover, am 2. Mai 1856. In: Karmarsch, Schule (wie Anm. 28), S. 209-212; vgl. auch die heutige studentische Interpretation auf https://www.geschichte.kit.edu/2008.php (15.1.2021).

30 Hier und im Folgenden Buresch, Feier (wie Anm. 28), S. 192 f. 
Sowohl in Prag als auch in Hannover zeigt die Aktivität in der Festvorbereitung, wie das Ereignis „Jubiläum“ vorausgenommen wurde. Als Vergangenheit, Gegenwart und Zukunft verbindendes Geschichtshandeln wurde es als eine Chance wahrgenommen, die genutzt aber eben auch verpasst werden konnte.

\section{Multimediale Geschichtsvermittlung}

Wenn bereits in der Jubiläumsplanung etwa Briefe oder Zeitungsannoncen als mediale Strategien eingesetzt wurden, um nach außen Relevanz zu behaupten, so wurden die Feiern selbst erst recht zur Plattform für die multimediale Kreation und Vermittlung von institutioneller Gravitas und Geschichtsbildung. Die in den drei jubilierenden Polytechnika eingesetzten Medien ähnelten sich zum Teil, unterschieden sich aber auch. Alle der betrachteten Jubiläumsfeiern waren durch eine Feststunde gekennzeichnet, in der Redebeiträge des Anlasses zur Feier gedachten. An allen Standorten wurden Publikationen herausgegeben, und in allen Jubiläen wurde versucht, aktive und ehemalige Schüler einzubinden und möglichst viele Alumni namhaft zu machen. Weitere Komponenten waren Gottesdienst, Umzug, Bankett, Ball, Kommers, Ehrung, Besichtigungen, Exkursionen und auch das Zusammenkommen in zwangloser Runde.

Die erste der drei Feiern veranstaltete das Dresdner Polytechnikum, das am 23. Mai 1853 sein 25-Jahr-Jubiläum beging. Das Datum der Feier war wie ein Spiegelbild des bescheidenen Anspruchs: Nicht der Jahrestag der Eröffnung (der 1. Mai) wurde zum Festtag gewählt, sondern die Geburtstags(nach)feier des sächsischen Königs Friedrich August II (1797-1854), wobei Hochschulfeiern zu Ehren der herrschenden Fürsten nicht unüblich waren. ${ }^{31}$ Auch das Festprogramm war genügsam: Es beschränkte sich auf eine Feierstunde mit ernsten und populären Gesängen des Schülerchors und den Reden des Polytechnikum-Direktors Julius Ambrosius Hülsse (1812-1876) und des königlichen Kommissars der Anstalt Christian Weinlig (1812-1873). ${ }^{32}$ Im Anschluss öffnete das Polytechnikum seine Sammlungen für das Publikum. ${ }^{33}$

31 Pommerin, Geschichte (wie Anm. 17), S. 47-49; vgl. Einladungsschrift der Königl. polytechnischen Schule in Stuttgart zu der Feier des Geburts-Festes Seiner Majestät des Königs Wilhelm von Württemberg, den 27. September 1849. Mit einer Abhandlung von J. M. Mauch. Stuttgart [1849].

32 Hülsse, Julius Ambrosius: Die Königliche Polytechnische Schule (Technische Bildungsanstalt) zu Dresden während der ersten 25 Jahre ihres Wirkens. Dresden 1853.

33 Hülsse, Schule (wie Anm. 32), S. 3. 
Die Ansprachen bilden dabei eine Geschichtssorte, die Vergangenheit und Gegenwart verknüpfte und aus gegebenem Anlass auch dem eigentlichen Geburtstagskind - nämlich dem Sachsenkönig - huldigte. Denn der seit 1850 mit der Direktion beauftragte Hülsse beschrieb eine Aufstiegsgeschichte der Anstalt, die Friedrich August II „aus einem schwachen Anfang zu der gegenwärtigen Entwicklung“ geführt habe. ${ }^{34}$ Auch Aufseher Weinlig erzählte im Stile eine Entwicklungsromans von der Reifung eines Jünglings, „so feiern wir auch mit vollem Rechte den heutigen Tag als Fest der Mündigkeit unserer polytechnischen Schule“. ${ }^{35}$ Weinlig war sich dabei des „vielleicht triviale[n] Vergleich[s]“ bewusst, strapazierte die Metapher aber trotzdem konsequent und zeichnete das Bild einer klein und schwach geborenen Institution, die nun aber „bereit und gerüstet [ist,] mit ihrem neuen Namen in der Reihe der gleichartigen Anstalten des deutschen Vaterlandes ebenbürtig zu kämpfen.“36

Beide Ansprachen verwiesen direkt auf gegenseitige und gegenwärtige Ansprüche. Mit der Betonung der Bedeutung des Herrschers für die Polytechnische Schule nahm Hülsse ihn in die Pflicht und ließ durch die langen Zahlenreihen zur pekuniären Ausstattung der Schule keine Interpretationsspielräume offen, was er sich auch in der Zukunft vom Fürsten erhoffte: „Die Fürsorge, welche von Sr. Majestät erleuchteten Regierung für die Beförderung wahrer Bildung ausgeübt wird, lässt sich nicht deutlicher als durch die eben ausgesprochenen Zahlen darlegen."37 Umgekehrt forderte das von Weinlig bemühte Narrativ des Heranwachsenden die politische Loyalität der Polytechniker ein und verbat jedes Flegelverhalten. Nur wenige Jahre nach der Revolution von 1848/49, in der etwa in Wien drei Viertel der immatrikulierten Techniker sich der Akademischen Legion angeschlossen hatten, ${ }^{38}$ lobte der Festredner die gute Erziehung in Dresden als Grund, dass es unter den hiesigen Polytechnikern keine „moralische und politische Verirrung“ gegeben habe. ${ }^{39}$ Zugleich war damit auch die Wissensvermittlung direkt berührt, da Weinlig strenge Schulzucht einforderte und akademische Lernfreiheit als Gefahr für das Gemeinwesen verstand.

34 Hülsse, Schule (wie Anm. 32), S. 4.

35 Weinlig, Christian: Die Rede des Herrn Königl. Commissars lautete. In: Hülsse, Schule (wie Anm. 32), S. 17-19, hier S. 17.

36 Weinlig, Rede (wie Anm. 35), S. 17.

37 Hülsse, Schule (wie Anm. 32), S. 15.

38 Vgl. Treue, Wilhelm: Zur Frühgeschichte der technischen Lehr- und Forschungsanstalten bis zu ihrer Beteiligung an der Revolution von 1848/49. In: Geschichte als Aufgabe. Festschrift für Otto Büsch zu seinem 60. Geburtstag. Hrsg. von dems. Berlin 1988. S. 267-297, hier S. 279.

39 Weinlig, Rede (wie Anm. 35), S. 18. 
Die Presseberichterstattung zum Prager Festakt legt nahe, dass dieser am 10. November 1856 entsprechend der oben dargelegten Planungen verlief. Im Beisein des Statthalters Karl Mecséry de Tsóor (1804-1885) und des österreichischen Kultusministers Leopold von Thun (1811-1888) wurden drei Reden gehalten. Neben Direktor Lumbe sprach der Fabrikbesitzer Anton Richter (1810 - 1880), und ein Schüler der Anstalt hielt eine „Dankrede“. ${ }^{40}$ Offizielle Vertreter des Wiener Polytechnikums fehlten indes unter den Gästen: Erst nach dem Festakt schrieb der dortige Direktor Karl von Smola (1802-1862), nicht ein einziger Professor hätte „ohne Beeinträchtigung der dienstlichen Obliegenheiten beizuwohnen vermocht“..41 Damit entzogen sich die Kollegen dem performativen Akt einer Relevanzproduktion durch Geschichtspraktiken. Vielmehr mahnte die in der Restaurationsphase nach 1848/49 noch immer unter militärischer Aufsicht stehende Schwesteranstalt post festum, den Prager Kollegen möge es gelingen, „den bereits erworbenen Ruf der Lehranstalt fort und fort zu steigern“, was zumindest auch als Spitze aus der ungleich bedeutenderen Wiener Anstalt gelesen werden konnte.

Am aufwendigsten und ausgiebigsten wurde in Hannover gefeiert, und zwar vom Freitag, dem 2. Mai 1856, bis zum Montagmorgen, ,als die Sonne schon hoch stand“. ${ }^{42}$ Bereits am Donnerstag wurden alle ankommenden Züge von Polytechnikern begrüßt und die Gäste in der geschmückten Bahnhofshalle in Empfang genommen. Von den zahlreichen Geschichtspraktiken verdient der Festzug, mit dem am Freitag die dreitägigen Feierlichkeiten begannen, besondere Aufmerksamkeit. Hier wurde Geschichte auch räumlich in die Öffentlichkeit getragen: Nicht nur Banner und Embleme, sondern die Mitlaufenden selbst wurden zu Medien, die auf die Geschichte des Polytechnikums verwiesen. Zugleich wies der Festzug mit seinen 800 Teilnehmern in die Zukunft, denn durch die Verbindung von Wissenschaft, raffinierter Umzugstechnik und reichlich Personal wurde zugleich der potenzielle zukünftige Beitrag des Polytechnikums in die Stadt und nicht zuletzt vor den Monarchen getragen.

Ein Herold führte den Festzug an, sodann wurde das Banner der Polytechnischen Schule gezeigt, „fünffeldig, mit den Landesfarben eingefaßt, im Mittelfelde als Sinnbild des Kampfes des Lichts mit der Finsterniß St. Georg, den Drachen besiegend, in den 4 umgebenden Feldern der pythagoräische Lehrsatz, Weltkugel, Zahnrad und Künstler-Wappen“. ${ }^{43}$ Eingefügt in eine 17 Glieder um-

40 O.V.: Nichtamtlicher Teil. In: Österreichisch-kaiserliche Wiener Zeitung (11.11.1856). S. 1044. 41 Hier und im Folgenden CVUT, Polytechnický ústav: Karl von Smola an Direktion Polytechnikum, 12.11.1856.

42 Karmarsch, Schule (wie Anm. 28), S. 205 f.

43 Hier und im Folgenden Buresch, Feier (wie Anm. 28), S. 195-199. 
fassende Ordnung folgten diverse weitere Einheiten, darunter zwei Musikkorps und fünf verkleidete vierspännige Festwagen. Sie repräsentierten Schwerpunkte der Lehre der Polytechnischen Schule, und das lange bevor sich das Abteilungswesen institutionell auch in Hannover durchsetzte. ${ }^{44}$ Die Wagen waren ausgeschmückt mit Insignien der jeweiligen Disziplinen sowie Medaillons ihrer lebenden und toten Heroen. Den betriebenen Aufwand zeigt etwa der Baldachin des Architektur-Wagens, der „einen durchbrochenen gothischen Thurmhelm nachahmend“ 24 Fuß in die Höhe ragte. ${ }^{45}$ Nur wenige Jahre nachdem sich die ersten Technikerverbindungen gebildet hatten, wurden sie in Hannover als Jubiläumsakteure in den Kreis der Geschichtsmächtigen einbezogen und konnten im Festzug ihre „schönen Fahnen“ präsentieren, Einheit mit ihren Lehrern inszenieren und akademischen Anspruch demonstrieren. Unter „Hoch“-Rufen verlief die Wegstrecke am königlichen Palais vorbei, wo sich König Georg V. (1819-1878) und die Königin Marie von Sachsen-Altenburg (1818-1907) „huldvoll dankend am Fenster“ zeigten.

An den Festzug schloss sich die Versammlung in der Aula des 1854 eingeweihten Lyzeums am Georgsplatz an - das Polytechnikum selbst verfügte lediglich über einen Hörsaal in dem auch im „Nothfall“ nur 120 Personen Platz fanden. ${ }^{46}$ Eingerahmt von Darbietungen des Polytechniker-Gesangvereins, der weitere Gedichte von Bibliothekar Rommel zu Melodien von Wolfgang Amadeus Mozart (1756-1791) und Ludwig Maurer (1789-1878) darbot, hatte die Festhandlung nur einen Tagesordnungspunkt: Ausführungen von Gründungsdirektor Karl Karmarsch, die „Thränen der Wehmut und Freude manchem Auge entlockten“. 47

Karmarsch nutzte in seiner Rede die Vergangenheit der Anstalt für in die Zukunft gerichtete politische Forderungen und postulierte die Ebenbürtigkeit von Polytechnika und Universitäten. Nicht nur verfügten auch erstere über akademische Lehrfreiheit. Mit den letzteren verband sie auch eine gemeinsame wissenschaftliche Grundlage ,in der Mathematik, dieser sichergehenden praktischen Zwillingsschwester der Philosophie." Und wie die Universitäten unterhielten auch die Polytechnika „ihre Fakultäten: des Bauwesens, des Maschinenwesens, des physikalisch-chemischen Faches.“ Forsch fuhr Karmarsch fort: „Sie haben, ohne

44 Vgl. dazu Nippert, Klaus: Wie entstand die Technische Hochschule? Zum Einfluss der Polytechnischen Schule Karlsruhe auf die Entwicklung eines Hochschultyps. In: Fridericiana 68 (2013). S. 9-16.

45 Hier und im Folgenden Buresch, Feier (wie Anm. 28), S. 199-201.

46 Karmarsch, Schule (wie Anm. 28), S. 101.

47 Buresch, Feier (wie Anm. 28), S. 201. 
Doktordiplome, ihre Doktoren“. ${ }^{48}$ Das fehlende Promotionsrecht über ein Paradoxon aufzulösen, war kühn und die darauf folgende Behauptung unhaltbar: „Die ganze Welt gibt zu, daß sich die technische oder realistische Bildung der so genannten gelehrten Bildung ebenbürtig an die Seite gestellt hat; neben dem Gymnasium geht die höhere Bürgerschule her; neben der Universität die polytechnische Schule, die ,technische Universität'. “ Tatsächlich ließ die Gleichstellung von Technischen Hochschulen und Universitäten noch Jahrzehnte auf sich warten. Die Mitte der 1850er Jahre erfundenen Jubiläen und die sie flankierenden Festschriften wurden früh zu einem Vehikel, diesen Anspruch zu untermauern, aber erst ab 1899 wurde den Technischen Hochschulen zunächst in Preußen durch den technikaffinen Wilhelm II. (1859-1941) zum Missfallen vieler Universitätsvertreter das Promotionsrecht verliehen. ${ }^{49}$

Das weitere Festprogramm sah ein Abendessen mit über 600 Personen im Lokal des Thalia-Vereins vor, das mit den Bannern des Festzugs geschmückt worden war. Die Performativität der Feier über die Region hinaus verdeutlicht ein Depeschenwechsel mit Christiania (dem heutigen Oslo), in dem innerhalb weniger Stunden Glückwünsche „von sieben norwegischen Freunden, ehemaligen Schülern der Anstalt“, das Antwortschreiben aus Hannover sowie der Empfang desselben ausgetauscht wurde. ${ }^{50}$ So wurde nicht nur die Telegrafie als Sinnbild technischer Errungenschaften gefeiert, sondern die durch Techniker entwickelten medialen Möglichkeiten genutzt, um Zusammengehörigkeit der Feiernden vor Ort und den Gratulanten im Ausland zu stiften. ${ }^{51}$

Die zentrale Figur des Jubiläums blieb Karl Karmarsch, da er als allzeitiger Direktor die Gründung und Entwicklung der Anstalt verkörperte. Die Verkündung, „daß die Philosophische Fakultät der Universität Göttingen dem verehrten Lehrer das Ehren-Doktor-Diplom verliehen“ hatte, ${ }^{52}$ führte zu großem Jubel, war sie doch über den Umweg der Person Karmarsch auch eine akademische Anerkennung seiner Schüler (und ihrer Institution) und zugleich eine Inversion der Praxis, als feiernde Anstalt Ehrungen zu vergeben. ${ }^{53}$

48 Hier und im Folgenden Karmarsch, Schule (wie Anm. 28), S. 218f. [Hervorhebung im Original]. 49 Vgl. Dietz, Burkhard, Michael Fessner u. Helmut Maier: Der „Kulturwert der Technik“ als Argument der Technischen Intelligenz für sozialen Aufstieg und Anerkennung. In: Technische Intelligenz und „Kulturfaktor Technik“. Kulturvorstellungen von Technikern und Ingenieuren zwischen Kaiserreich und früher Bundesrepublik Deutschland. Hrsg. von dens. Münster/New York 1996 (Cottbuser Studien zur Geschichte von Technik, Arbeit und Umwelt 2). S. 1-32.

50 Buresch, Feier (wie Anm. 28), S. 203.

51 Logge, Thorsten: Zur medialen Konstruktion des Nationalen. Die Schillerfeiern 1859 in Europa und Nordamerika. Göttingen 2014. S. 169.

52 Buresch, Feier (wie Anm. 28), S. 202.

53 Müller, Inszenierte Erinnerung (wie Anm. 2), S. 82. 
Nachdem am Samstagvormittag die Sammlungen des Polytechnikums zu besichtigen waren und Exkursionen $\mathrm{zu}$ „technisch interessanten Baulichkeiten, Fabriken etc. “ durchgeführt wurden, ${ }^{54}$ fand am Samstagabend ein Kommers statt. Die Überlassung des zweiten Festabends an die Lernenden entsprach einer weiteren Übernahme universitärer Jubiläumstradition und war beredter Ausdruck der innerinstitutionellen Anerkennung der Schüler durch die Lehrenden. Auffallend ist, dass der Sonntag nicht wie in Prag für einen Festgottesdienst genutzt wurde die inszenierte Dankbarkeit anlässlich des Jubiläums der Hannoverschen Polytechniker war weltlich konnotiert. Ein Ball am Abend des 4. Mai 1856 beschloss das Fest.

Eine weitere Ehrung, die Karmarsch allerdings erst ein Jahr später in Empfang nehmen konnte, erhielt er von den Schülern: einen knapp $70 \mathrm{~cm}$ großen PrunkPokal. Er verdeutlicht auf besondere Weise die cross- und multmediale Dimension des Hannoverschen Jubiläumshandelns auch jenseits der nachhaltig dominierenden textuellen Erzählung. Der Pokal zeigt sechs Bildnisse, die zugleich als Allegorien auf die am Polytechnikum vermittelten Künste zu lesen waren, und am oberen Rand der Schale verläuft eine Darstellung des Jubiläumsfestzugs. Eingerahmt von dem Festzug steht die Inschrift: „Dem ruhmgekrönten Förderer technischer Bestrebungen, dem treuen Lehrer die dankbaren Polytechniker“. Das metallene Prunkgefäß war nach der Zeichnung des Architekten Konrad Wilhelm Hase gefertigt, und Karmarsch mit seinem Gespür für (geschichtsbasierte) Öffentlichkeitsarbeit überführte dieses Bildnis wiederum zurück in eine zweidimensionale Medialität:

„Ich ließ den Pokal photographieren, nach der Photographie eine lithographierte Zeichnung machen, setzte unter diese einige autographierte Widmungsworte mit meiner Unterschrift, und ließ Abdrücke allen Polytechnikern zukommen.“55

\section{Die Festschriften und die Modi der Relevanzzuschreibung}

Aufgrund ihrer Anlage einer nacheinander geschalteten Kommunikation zählen dickleibige Festschriften $\mathrm{zu}$ den nachhaltigsten Ereignissen eines Hoch-

54 Buresch, Feier (wie Anm. 28), S. 204.

55 Karmarsch, Karl: Ein Lebensbild, gezeichnet nach dessen hinterlassenen „Erinnerungen aus meinem Leben“. Mit Ergänzungen von Egb. Hoyer. Hannover 1880. S. 141. 


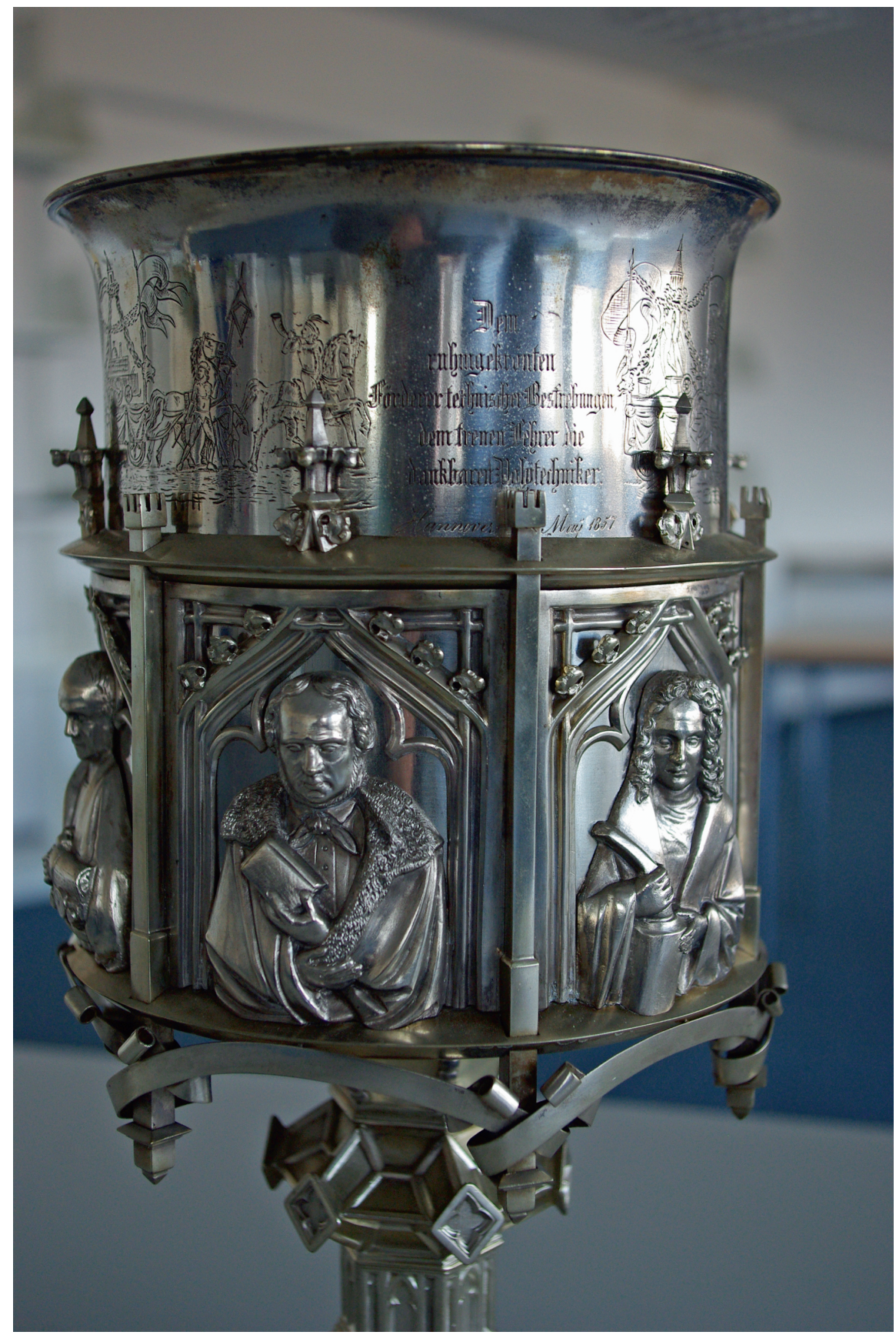

Abb. 2: Der Ehrenpokal für Karl Karmarsch zeigt den Jubiläumsfestzug (Konrad Wilhelm Hase, 1857). 
schuljubiläums. ${ }^{56}$ Doch trotz der klassischen, Wissen aufbewahrenden Anlage von Büchern, können auch sie genutzt werden, um nicht nur die Vergangenheit, sondern auch die Gegenwart von Institutionen performativ zu (re-)konstruieren. Das Dresdner Polytechnikum legte die knappste Publikation vor: einen 54-seitigen großformatigen Band, dessen Hauptzweck die Dokumentation der Feier durch den Abdruck der Reden war. Durch elf Beilagen, in denen die Entwicklung der Anstalt durch Aufstellungen zu den bisherigen Schülern und Lehrern, Kursen und Stipendien dokumentiert wird, erhielt die Publikation zugleich Historiographie-, bzw. Chronik-Charakter.

Das Polytechnikum in Prag publizierte dagegen eine umfassende monographische Chronik. ${ }^{57}$ Wenngleich der Umfang das ursprünglich Geplante übertraf, war sie von Anfang mit einem hehren Ziel angelegt. Sie sollte, um „der vaterländischen Kulturgeschichte Rechnung zu tragen“, die Geschichte der Anstalt und ihrer Mitwirkenden „der Vergessenheit [...] entreißen“. ${ }^{58}$ Anders als bei den anderen hier betrachteten Festpublikationen zeichnete nicht der Direktor, sondern ein weiteres Mitglied des Lehrkörpers, nämlich der Mathematiker, Astronom und Meteorologe Carl Jelinek (1822-1876) dafür verantwortlich. Den größten Abschnitt bildete ein Abriss der Geschichte und Vorgeschichte der Anstalt, der vom Umfang her zweite Posten war ein namentliches Verzeichnis einiger ehemaliger Prager Polytechniker und ihres nunmehrigen Berufsfeldes. Hinzu kommen biographische Notizen zu ehemaligen und aktiven Lehrern, Berichte über die Sammlungen und Auswertungen zur Schüler-Frequenz.

Das in Hannover herausgebrachte Werk war besonders penibel orchestriert worden. Die Festschrift verband den umfänglichen Charakter der Prager Darstellung mit der Dokumentation der Feier, wie es auch die Dresdner Anstalt für ihren Festakt vorgemacht hatte. Die Festschrift konnte auf der regen Publikationstätigkeit von Direktor Karmarsch aufbauen. So erschien das Jubelbuch unter dem schlichten, aber gleichzeitig den Anspruch auf Gegenwartsrelevanz durch Nachführung demonstrierenden Titel „Die polytechnische Schule zu Hannover, zweite sehr erweiterte Auflage“. ${ }^{59}$ Dazu passte, dass die Festschrift zunächst das aktuell geltende Regelwerk bis hin zum Schulgeld darstellte und damit dezidiert

56 Nicolaysen, Rainer, Eckart Krause u. Gunnar B. Zimmermann: Einleitung. In: 100 Jahre Universität Hamburg. Studien zur Hamburger Universitäts- und Wissenschaftsgeschichte in vier Bänden. Bd. 1: Allgemeine Aspekte und Entwicklungen. Hrsg. von dens. Göttingen 2020. S. 9-30, hier S. 10.

57 Jelinek, Carl: Das ständisch-polytechnische Institut zu Prag. Programm zur fünfzigjährigen Erinnerungs-Feier an die Eröffnung des Institutes. 10. November 1856. Prag 1856.

58 CVUT, Polytechnický ústav: Lumbe an Ständischen Landesausschuss, 17.6.1856.

59 Karmarsch, Schule (wie Anm. 28). 
eingeschriebene und prospektive Schüler adressierte. Erst auf diesen praktischen Teil folgten historische und hagiographische Kapitel wie die 20-seitige Chronik, ein 30-seitiger „Rechenschaftsbericht über Wirksamkeit und Erfolge der polytechnischen Schule während der ersten 25 Jahre ihres Bestehens“ und die 40seitige Dokumentation der Feier. Dabei sorgte nicht zuletzt der Sprecherwechsel dafür, dass auch die „Hoch!“-Rufe auf Direktor Karmarsch ohne größere Peinlichkeit mitgeteilt werden konnten. ${ }^{60}$

Zudem wurden auf etwa 80 Seiten die ehemaligen Schüler sowie ihre letzte bekannte berufliche Position wiedergeben. Die wesentlichen Berufszweige der 1272 ermittelten Alumni waren Fabrikanten und Handwerker (182), das Bauwesen (173), die Landwirtschaft (158), die Landvermessung (95), das Eisenbahnwesen (91), das Militär (80), der Maschinenbau (78), das Lehrfach - inklusive Studierende (70), Gewerbliche Künstler (69), das Forstfach (64) und der Handelsstand (52). ${ }^{61}$ Diese Zahlen deuten auf eine gewisse Bedeutung der Anstalt für die Industrialisierung des Königreichs Hannover hin; eine allgemeine Einordnung der Wirkungsgeschichte der Polytechniker und ihres sozialen Hintergrunds bedarf weiterer Forschung. ${ }^{62}$

Nicht nur in Hannover, sondern auch anlässlich der anderen hier untersuchten Jubiläen wurde über Zahl und Wirken der Alumni gesellschaftliche Relevanz proklamiert. Die Polytechnika lieferten - so die Dresdner Interpretation das „Samenkorn wissenschaftlicher Auffassung, das durch die befruchtende Berührung mit dem mannichfaltigen Erscheinungen des Berufslebens keimt“, sich aber „der statistischen Erörterung durch Zahl und Mass“ entziehe. ${ }^{63}$ Die Messbarkeit der Relevanz der Schule wurde somit ins Ungefähre verschoben und zugleich ein Anteil am allgemeinen Fortschritt für sich reklamiert: Denn „dass ein Theil dieser Erfolge Wirkung unserer Bildungsanstalten und speciell der polytechnischen Schule“ sei, konnte damit „wohl unbedingt behauptet werden.“ Diese Behauptung untermauerte Hülsse durch detaillierte Angaben etwa zum Schulbesuch und zu den belegten Fächern. Insgesamt hatten über 2.100 Schüler die Dresdner Anstalt zumindest für einige Zeit besucht, die knapp 270 Absolventen der unteren Abteilung und Besucher der oberen Kurse wurden namentlich benannt.

60 Buresch, Feier (wie Anm. 28), S. 202.

61 Karmarsch, Schule (wie Anm. 28), S. 180 - 187.

62 Einen eher nachrangigen Anteil der Polytechnika an der Industrialisierung vermutete Wolfgang König, vgl. König, Verwaltungsstaat (wie Anm. 11); vgl. zu Hannover Mundhenke, Herbert: Die Matrikel der Höheren Gewerbeschule, der Polytechnischen Schule und der Technischen Hochschule zu Hannover. Hildesheim 1988.

63 Hier und im Folgenden Hülsse, Schule (wie Anm. 32), S. 16. 
Auch in der Prager Darstellungen nimmt der Verweis auf die eigenen Schüler eine zentrale Funktion ein. Von den etwa 12.000 ehemaligen Schülern meldeten sich auf die Zeitungsannoncen binnen weniger Wochen immerhin 1668 Alumni, die vorrangig im Bauwesen (527), im Eisenbahnwesen (321) und im Vermessungswesen (274) Anstellung gefunden hatten. ${ }^{64}$

Die Hannover und die Prager Darstellung zeigen zudem, dass zeitgenössische Jubiläumsgeschichtsschreibung Relevanz auf subtilere Art generiert als es der Abdruck von Schülerverzeichnissen und Jubiläumsreden vermag. Die Polytechnika wurden gegründet, um die regionale Industrie über Bildung zu befördern und - das findet sich explizit in Prag als weiterhin relevantes Motiv - die Abhängigkeit vom Ausland zu reduzieren. Dass dies in Prag noch immer pressierte, war eigentlich ein Eingeständnis, auch in 50 Jahren den Gründungszweck nicht erreicht zu haben. Da die drängende Neuorganisation im Zuge der Revolution von 1848 abgebrochen wurde und das Institut seit fast einem Jahrzehnt nur eine provisorische Leitung hatte, präsentierte Chronist Jelinek anstelle einer Hagiographie eher ein Klagelied, das zum Ende die drückende Gegenwart ansprach und die zurückliegende Unterstützung als Verpflichtung für die Zukunft wendete.

\begin{abstract}
„Der hochl. Landesausschuß und das h. Unterrichtsministerium, welchen die Prager Anstalt ihre Gründung und so vielfache Bereicherungen und Erweiterungen verdankt, werden ihr Werk krönen durch die Neugestaltung der ältesten technischen Schule in Österreich, welche jedes auf sie verwendete Opfer durch ihre Zöglinge zehnfach wieder ersetzen wird, denn wohl ist ein Capital zu guten Zinsen angelegt, welches die technische Bildung zu fördern bestimmt wird [...]!“65
\end{abstract}

Dagegen schreckten die Autoren der Hannoverschen Festschrift auch vor deutlichem Eigenlob nicht zurück, als sie das Wirken der Lehrer in umfänglichen Personalnotizen akzentuierten und deren Bedeutung als öffentliche Gutachter unterstrichen. ${ }^{66}$

Wie in der Vorbereitung und Durchführung der Jubiläumsfeierlichkeiten so wurden auch in den Festschriften die Bedeutung der gefeierten Anstalten durch die Verschränkung von Vergangenheit und Zukunft in der Gegenwart hervorgehoben. Dies gelang sogar dann, wenn die Gegenwart als problematisch galt. Jelinek hoffte für das Prager Polytechnikum, „daß der gegenwärtige bedeutungsvolle Moment, die Veranlassung geben möge, die Frage der Neugestaltung [...] zum baldigen Abschluß zu bringen. ${ }^{“ 67}$ Die Reform ließ indes weiter auf sich

64 Jelinek, Institut (wie Anm. 57), S. 259

65 Jelinek, Institut (wie Anm. 57), S. 116.

66 Karmarsch, Schule (wie Anm. 28), S. 188.

67 Jelinek, Institut (wie Anm. 57), S. 115. 
warten, und sein seit 1849 am Polytechnikum tätiger Kollege Carl Kořistka (18251906) musste Anfang der 1860er Jahre (erneut!) auf einer Rundreise die europäischen Polytechnika als Vorbilder inspizieren. ${ }^{68}$

In den Festschriften aus Dresden und Hannover wurde die Temporalität der Feier selbst durch den wortgetreuen Abdruck von Einschüben in den Reden - zum Beispiel in der direkten Ansprache an die Gäste, die die Anstalt „heute mit ihrer Gegenwart beehrten“ - festgehalten. ${ }^{69}$ Dabei wurde die inzwischen vergangene Gegenwart des Feierns selbst mit der gefeierten Vergangenheit verbunden und zugleich mit der Zukunft verknüpft, da das „Bestehen noch den Generationen später Jahrhunderte zu Statten kommen soll."70 Deutlich zeigt sich die Konvergenz von Vergangenheit, Gegenwart und Zukunft in Karmarschs Formulierung von den „ersten 25 Jahre[n]“ der Hannover Anstalt, ${ }^{71}$ während Weinlig die gegenwärtige Bedeutung des Dresdner Polytechnikums durch das zukünftige Wirken der Schüler begründete: „Wir vertrauen Ihnen, geliebte Zöglinge der Anstalt, das Theuerste auch fernerhin an, was wir haben, den Ruhm unserer polytechnischen Schule; bringen Sie denselben zu immer erhöhter Geltung!“72

Trotz des selbstverständlichen Ausgriffs auf Vergangenheit und Zukunft schien gerade die Gegenwart der 1850er Jahre von besonderer Qualität. Sie wurde als eine nur selten in Jahrtausenden vorkommende Phase des technischen Fortschritts beschrieben. Daher sei auch die Feier einer höheren technischen Bildungsanstalt kein „gewöhnliches Vergnügungsfest“, sondern „die Verherrlichung einer großen und mächtigen Sache, einer urkräftigen Äußerung des Weltgeistes [...], deren Entstehung, Wachsthum und Blüthe zu sehen unserer Zeit vorbehalten war". ${ }^{73}$

\section{Ausblick: Alumni als Träger polytechnischer Jubiläumskultur}

Die Jubiläen der drei Polytechnika Dresden, Hannover und Prag waren Anlass, zahlreiche Geschichtssorten zu produzieren, um mit unterschiedlichen medialen Strategien Aufmerksamkeit für die Anstalten zu generieren, Relevanzbe-

68 Kořistka, Carl: Der höhere polytechnische Unterricht in Deutschland, in der Schweiz, in Frankreich, Belgien und England. Gotha 1863.

69 Hülsse, Schule (wie Anm. 32), S. 13.

70 Karmarsch, Schule (wie Anm. 28), S. 216.

71 Karmarsch, Schule (wie Anm. 28), S. 158.

72 Weinlig, Rede (wie Anm. 35), S. 17.

73 Buresch, Feier (wie Anm. 28), S. 206. 
hauptungen aufzustellen und Zukunft zu gestalten. Die Polytechnika nutzten dabei Publikationen heterogener Ausrichtung und verschiedene performative Akte, um sowohl die Angehörigen der Institutionen als auch Externe der Bedeutung der Einrichtungen zu versichern und um die weitere Förderung durch die übergeordneten Behörden und der Fürsten sicherzustellen. Dabei übernahmen die Polytechnika in vielen Fällen Jubiläumspraktiken von Universitäten. Der explizite Anwendungsbezug der Polytechnika führte allerdings auch zu neuen und spezifisch „polytechnischen“ Formen des Jubiläumshandelns. Insbesondere der Versuch, über das Jubiläum mit Gewerben und Handel in Kontakt zu treten, fällt hierbei ins Auge. Zentral war dabei der Konnex über die ehemaligen Schüler, und die Kontaktaufnahme im Vorfeld der Jubiläen zeigt sich als wichtiger Baustein im Jubiläum und als eine eigene Form individualisierter und zugleich institutionalisierter geschichtsbasierter Öffentlichkeitsarbeit.

Nicht nur die Direktoren und weitere aktive Lehrer wurden zu Jubiläumsakteuren. Die Schüler wirkten an allen drei Einrichtungen an den Feierlichkeiten mit. Der Auftritt der Verbindungen in Hannover zeigt dabei nicht nur die interne Teilhabe unterschiedlicher Gruppen, sondern ist zugleich Inszenierung einer „universitas magistrorum et scholarium“ und damit Ausweis des Anspruchs auf Gleichrangigkeit der Polytechnika mit den Universitäten. In Dresden dagegen zeigten sich im Jubiläum diametral davon abweichende Vorstellungen über die innere Ausgestaltung der Institution, die Rolle von Lehrenden und Lernenden und über die Modi der Wissensmehrung und -vermittlung.

Die betrachteten Jubiläen korrespondierten miteinander, etwa als Vorbilder oder durch gegenseitige Gratulationen. Die Verschränkung der Jubiläen (über die Ehrenpromotion von Karmarsch auch mit dem Universitätssektor) führt zu weiterführenden Fragestellungen. Insbesondere der Brückenschlag zu Wirtschaft und Alumni gehört mittlerweile zum festen Bestandteil vieler Hochschuljubiläen, sodass nicht nur die weitere Entwicklung der Feiern an den Technischen Hochschulen, sondern auch die Übernahme ihrer spezifischen Jubiläumsmodi durch die „alten“ Universitäten lohnende Forschungsfelder darstellen. Hierbei bieten sich auch Festschriften als Quelle an, gerade im Falle fehlender Aktenüberlieferung. Den Quellenwert dieser Publikationen zeigen nicht zuletzt die Erhebungen zum Verbleib der Polytechniker, deren Anteil an der im internationalen Vergleich nachholenden Industrialisierung Deutschlands ein drängendes Desiderat darstellt, das künftig auch über KI-gestützte prosopografische Forschung aufgehellt werden könnte. ${ }^{74}$

74 Vgl. Hawicks, Heike u. Ingo Runde (Hrsg.): Universitätsmatrikeln im deutschen Südwesten. 
Auch die temporale Offenheit von Jubiläumshandeln lässt sich gut mithilfe der Festschriften untersuchen, die in Dresden und Hannover bewusst dazu genutzt wurden, die flüchtigeren Geschichtsinszenierungen ,aufzubewahren“. Hier zeigt sich ein Bewusstsein für die Gegenwart als künftige Vergangenheit, deren Deutung geprägt werden sollte. Diese „Futur II-Brille“ erwies sich als wirksam, und das Geschichtshandeln der technischen Lehranstalten in der Mitte des 19. Jahrhunderts ist als Erfinden von Traditionen zu verstehen. ${ }^{75}$ Nicht umsonst galt die Prager Festschrift aus dem Jahr 1856 noch 50 Jahre später als der Maßstab für das Folgeprojekt, mit dem die nunmehrige deutsche Technische Hochschule in Prag auf ihr erstes Säkulum zurückblicken wollte. ${ }^{76}$

Bestände, Erschließung und digitale Präsentation. Heidelberg 2020 (Heidelberger Schriften zur Universitätsgeschichte 9).

75 Vgl. auch Albrecht, Bergakademie (wie Anm. 3), S. 78.

76 Stark, Franz (Red.): Die k.k. deutsche technische Hochschule in Prag 1806-1906. Festschrift zur Hundertjahrfeier [Vorwort]. Prag 1906. 
